\title{
Erratum to: Excitons in ZnO Quantum Wells
}

\author{
M. N. Bataev ${ }^{a}$, N. G. Filosofov ${ }^{a}$, A. Yu. Serov ${ }^{a}$, V. F. Agekyan ${ }^{a}$, \\ C. Mohrain ${ }^{b}$, and V. P. Kochereshko ${ }^{a, c}$, * \\ ${ }^{a}$ St. Petersburg State University, St. Petersburg, 199034 Russia \\ ${ }^{b}$ Centre de Recherche sur I'Hetero-Epitaxie et ses Applications-CNRS, F-06560 Valbonne, France \\ ${ }^{c}$ Ioffe Institute, St. Petersburg, 194021 Russia \\ *e-mail: Vladimir.Kochereshko@mail.ioffe.ru \\ Received February 20, 2019
}

DOI: $10.1134 / \mathrm{S} 106378341903034 \mathrm{X}$

The name of the fifth author should read Mohrain.

The original article can be found online at https://doi.org/10.1134/S1063783418120077. 